\title{
14. THE MANAGEMENT OF WORK FORCE IN NETWORKED ORGANIZATIONAL ENVIRONMENT
}

\author{
Lic.Sc. Jukka Viitanen \\ Turku School of Economics and Business \\ Administration \\ Institute of Information Systems Science
}

\begin{abstract}
This presentation focus on describing the management challenges of the work force in turbulent business environments, where corporations rely increasingly on interorgani-zational arrangements. The networked organizational environment has brought along novel requirements for extreme flexibility and openness in directing operations, and consequently, pose novel challenges in managing the human resource pool. These challenges are summarized into two broad categories: promoting cultural integration and ensuring continuous renewal. The integration promotion takes place in core processes for building up the shared social architecture and for realizing the coordinated cooperation. The continuous renewal, in turn, is managed through innovation and entrepreneurial empowerment processes. Based on this examination, we address the questions for the SIS role in supporting the organizational change process and in facilitating the efficient communication in cross-boundary relationships. In the final part, some recommendations are presented for the educational activities and for personnel training in organizations.
\end{abstract}

The original version of this chapter was revised: The copyright line was incorrect. This has been corrected. The Erratum to this chapter is available at DOI: 10.1007/978-0-387-35393-7_22 


\section{The Rapid Change in Competitive Environment is Posing New Pressures for Working Conditions in Organizations}

This presentation is based on a commonly perceived fact that business environment is changing in ever-increasing pace. Strong forces of chance like escalating customer diversity, blurring of market boundaries, deregulation and technological discontinuities (Cravens et al. 1994; Ilinitch et al. 1996; Prahalad-Hamel 1994) are reshaping the competitive landscape worldwide. As a result most companies are not only undergoing a rapid and radical structural change, but are also experiencing a fundamental shift in the rules of competition and the way the game of competition itself is played. This change process is seen irreversible in redefining the how and where the work is done and how it is organized (Scott Morton 1995). The once clearly defined boundaries of markets and organizations are becoming more intermingled and interorganizational dependencies change gradually the overall working conditions.

This hypercompetitive pressure (Hansen-Bauer-Snow 1996; Ilinitch et al. 1996), under which competitors act boldly and aggressively to disrupt the prevailing status quo, leaves but one choice for managers to radically reshape and redirect their organizations in their ability to counter the external threats and pressures. However, while it is still necessary to maintain some structure in organizing the corporate activities and in building shared visions and values in operations, the managerial equation for managing the work force remains extremely challenging.

Volberda (1997) has stated that in developing the corporate flexibility, we can identify both managerial and organizational design tasks. The flexibility is seen as a function of both the control capacity of the management and the changeability of the organization. It is defined as follows:

Flexibility is the degree to which an organization has a variety of managerial capabilities and the speed at which they can be activated, to improve the controllability of the organization under unexpected disturbance (ibid. 1996,361).

A firm is 'under control' when for each competitive change there is a corresponding managerial capability and firm response. In stable environments flexibility is not a serious strategic option to achieve control, but in hypercompetitive environments the required high responsiveness can be achieved only through an increased flexibility.

The flexibility focus must address to different levels of management action. The operational flexibility consists of routine capabilities that are based on present structures or goals of the organization. These routines are used primarily in reactive manner to provide rapid response to changes that are familiar or predictable. Examples are found in variation of production volume, building up the inventories and using temporary work force and multisourcing within the supplier network.

The structural flexibility is addressed in creating multifunctional teams and changing managerial roles to facilitate the renewal or transformation of current organizational processes. The external focus is on efficiency and effectiveness of corporate value activities (Porter 1985; Ohmae 1985) by reconfiguring the primary 
activities with stake holders, and industry wide coordination of activities to maximize the flexibility of the relevant industry value system.

The strategic flexibility is related to the goals and competitive moves of the corporation, and must be exercised in situations where management faces unfamiliar changes in environment that have far-reaching consequences for future successful operation. The most effective means are usually some fundamental change/changes in products or processes, application of newest technologies, or creating new product market combinations for competitive control. This most radical type of flexibility is much more qualitative and involves changes in the nature of organizational activities. The creation of new activities in new situations have proved to be important, while in this way the management can commit to an ongoing process of self-renewal and continuous adaptation (Theuerkauf 1991; Hansen-Bauer-Snow 1996). In turbulent environments, this kind of foundation have high tolerance of ambiguity, which is to be promoted by encouraging employees for innovative thinking, entrepreneurship, and self-empowerment, but also maintaining a balanced base in shared values and goals.

As can be seen, the fundamentals for organizing the work force in networked environments differ noticeably from the traditional organizational settings. The requirements for changeability of the organization and flexibility in organizing the work force itself have emerged as the key factors in determining the future operational and strategic success. The organizational renewal and continuous adaptation to incremental changes in business process call for high tolerance for ambiguity and direct management to enforce employees' innovative thinking and self-empowerment. The employees, in turn, should accept both the use of temporary work force and the vague job descriptions, to secure organization's adaptability to change.

\section{The Human Resource Management Challenges in Networked Organizations}

The networked organizations are highly dependent on their supporting communication infrastructures, while information sharing takes place primarily in connecting physical networks. Thus, the corporate systems architecture is seen as a necessary backbone for interorganizational cooperation. However, it should be noted that this architecture is only a technical enabler in networking process and, as such, remains in a supportive role in explaining the challenges for the actual human resource management.

In networked organizations, the management's attention is focused to the human resource and relationship assets, which are the two key areas for building up the organizational capability (Ross et al. 1996; Manheim 1992). The development of human resource assets is concentrating on motivating and skilling personnel to solve business problems, and aim at developing individual competency on information access and use. The specific IS-related focus is, thus, on boosting ISrelated learning and on pooling efficient human resources with SIS (Broadbent et al. 1996). The network must be managed as a combination of both human systems and technology, which evolve in dynamically changing business environments. 
While the managerial challenge is to promote integration by providing a basis for shared social architecture and coordination for networked cooperation (Ching et al. 1996; Bartlett-Ghoshal 1993), the management of relationships assets focuses on developing excellence in communication. This focus is closely related to cultural questions both within the given network and in its environment. In this, the connecting infrastructure can support the required high-order stable characteristics of corporate culture, norms etc. by defining the 'degree of belonging' of each individual member through shared understanding of their relative importance and roles in any given network (Hagström 1991). The objective is to create a socially motivating and equal culture for intranetwork collaboration.

To gain collaborative advantages from this collaboration, network members ought to remember at least the following two fundamentals of alliancing and networking (Kanter 1994; Johnston-Lawrence 1988; Lei 1993):

- they cannot be "controlled" by formal systems, but require a dense web of interpersonal connections and internal infrastructures that enhance learning and information sharing, and

- the partners should develop linkages and shared ways in operations so that they can work together smoothly, finally institutionalizing the informal arrangements.

This means that each partner should engage in their shared vision and be ready to invest time and resources in their strategic alliance. They have to behave in an honorable way that justifies and enhances mutual trust, and not to be the first ones to play games at an ally's expense. However, in a network relation the players should be aware of the fact that collaboration is just competition in a different form (Hamel et al. 1989; Jarillo 1988).

The management should emphasize the importance of middle- and lower-level personnel in interfunctional and interorganizational dialogue rather than promoting a rigid top management control and guidance. In this, they should rely heavily on lateral communication instead of vertical dialogue, as well as determine network center as an architecture of communication infrastructure and shared vision, and a protector of knowledge investments in creating organizational capabilities (Hedlund 1994).

In networked organizations, the management focus tend to both narrow and broaden simultaneously. From one point of view, they encourage unbundling and outsourcing of undifferentiated value activities, and from other, expand from maximizing value for an individual corporation to maximizing value for the network as a whole (Hagel III 1996). Accordingly, the human resource management challenges can be summarized into two broad categories: promoting integration and ensuring continuous renewal ${ }^{3}$. The integration promotion takes place in core processes for building up the shared social architecture and for realizing the coordinated cooperation. The continuous renewal, in turn, is managed through innovation and entrepreneurial empowerment processes.

${ }^{3}$ This division is adapted broadly from Bartlett-Ghoshal (1993), Charan (1991) and Kanter (1994). 
In building up the shared social architecture, managers must encourage the development of an organization-wide shared vision and personal commitment to jointly determined objectives. This notion emphasizes the need for more sophisticated coordination mechanisms, which can form a portfolio of coordinating processes that are used to cope with formal and informal role assignments as well as institutionalizing the chosen procedures and systems (Lorenzoni et al. 1995; Charan 1991).

The close inter-partner socialization can help individual members to learn, absorb, and internalize the tacit knowledge and skills possessed by their partners (Lei 1997; Culpan 1993). This process must be supported by extensive intranetwork training projects that are managed from the center. This is to promote the emergence of common concepts, terminology and cognitive maps, which it turn, facilitate the creation of commonly perceived internal culture (Jarvenpaa-Ives 1994; HanssenBauer-Snow 1996). Through these commonalities the network can 'walz to the same tune', which encourage joint decision-making, vertical and horizontal planning, and the fusion of competent allies despite their organization cultural differences.

The coordination of cooperation is addressed through synergy management and solutions for communication and information sharing. The overall network structure is syncronized so that individual units work united as a team of organizations and functional synergy potential can be realized.

The synergy management includes mechanisms for identifying the network affinity groups, quantification of the synergy potential and formulation of a clear horizontal strategy for network-wide cooperation (Vitzjak 1994). The network is organized around linked processes, where multiple competencies can be combined to maximize the synergy petential and transaction value both within and across the network boundaries. The technical excellence of individual members can be linked with complementing resources and, then, transferred and leveraged broadly across the entire process (Ostroff-Smith 1992). The cultivation of complementary skills and resources are brought under one united coordination process.

The networked organizations are building their core competence especially on the organizational capabilities and flexibility in international operations (PrahaladDoz 1989). Therefore, the management focus is on creating positive values, attitude, and behavior towards mutual information sharing, even between competing and collaborating partners. They also aim at developing individual competency related to pooled information use, and leveraging organization's knowledge with public information environment.

Networks must ensure that they maintain their flexibility and responsiveness in turbulent business environment. Consequently, the other broad management challenge is to ensure renewal through continuous innovation, while protecting the accumulated knowhow and organizational capabilities. For this purpose, the networked organizations usually rely on modular structure and changeable process capabilities. How then, the center can promote network's innovative-ness and knowledge transfer?

The management should focus their efforts on to constantly branching out of the new opportunities, to let the network members freely introduce new ideas in pursuing their business objectives (Ciborra 1996; Bartlett-Ghoshal 1993). The 
center can set up learning races, where partners are given a common goal with a prize for first to achieve the target. Usually this prize in the opportunity to lead off the exploitation of the invented new development, which in turn, may lead to coventuring or internal "intrapreneurship" for future financial benefits (Miles-Snow 1986). The center may act as an innovation market place, where the overall innovation process of the network takes place. In this position, it can both acquire and extend its own competencies as well as promote the network renewal.

The fourt management area is found in empowering the network for entrepreneurial activity. This is promoted by assigning an owner to each individual process and by providing participating members a chance for personal or corporate specific rewards (Ostroff-Smith 1992; Cravens et al. 1994; Jarvenpaa-Ives 1994). This empowerment is, in part, supported with all the above management activities: by creating an appropriate social infrastructure for shared responsibility, promoting investments, and providing the tools for both communication and information sharing. Thus, each member can be equipped for entrepreneurial initiatives. However, since the front line network members decide autonomously how to pursue these opportunities, the final results will emerge only out of free will and in an atmosphere of shared responsibilties. It is also argued, that the assignment process must be realized by protecting simultaneously both personal freedom and privacy.

\section{Managerial Action Plan for Enhancing Networking and Improved IS-Related Training for Networked Organizations}

In networked environments, communication across organizational borders is the descriptive element of all business activities. For information management (IM), this changed reality means a novel challenge to find common elements across the network as a whole. The IM focus is essentially in gathering, storing and disseminating business information to generate multidimensional knowledge valued by customers or alliancing business partners (Jarvenpaa-Ives 1994; Ghoshal et al. 1994). This knowledge should be available and accessible to any person, who comes in contact with the network, in any form, at any time and in every location. The joint dynamic operations call for flexible architectures with open access to all network information. These demanding requirements create obvious challenges for the interorganizational information management which can be summarized as follows (Jarvenpaa-Ives 1994; Teramoto-Iwasaki 1991):

- building a flexible and efficient information architecture across knowledge nodes located either in headquarters or in subsidiaries,

- creating the values, attitudes, and behaviors of mutual information sharing, even between competing and collaborating partners,

- building applications and databases around network relationships approach

- creating understanding for the mobile and culturally diverse workplace, 
- developing individual competency related to transnational information use,

- leveraging and interfacing with public information environment, and

- protecting simultaneously personal freedom and privacy.

As can be seen, these information management challenges combine both organizational and technical IS management within the same frame. It emphasizes the needs for integration and coordination on the global scale especially in human resource and alliancing relationships fields. The technical element, in turn, is seen as an enabler for this interaction.

Several trends in IS/IT industries have supported the emergence of networking. The rapid advancement of the information technology itself, the rise of network computing, and the growth of IS service segments have proved to be providing an alternative to the traditional transaction processing solutions inside and between organizations (Markus 1996). However, the technology development must be balanced with organizational processes to guarantee a possible realization of the IS/IT-related benefits. These notions determine the main challenge for managers in IM function; to create a cohesive organizational climate for future application and architecture development.

In terms of integration promotion the education and development challenge in IS field is in finding the "right tune" for the formerly separated domains of business and information function. We need to consider the underlying elements of internal network relationships and organizational culture. A technological infrastructure and solutions can be used to connect people and information, but only those organizational settings that favor change sensitive operational logic can adapt flexibly and efficiently to the challenge of turbulent environment. It has also been argued that cultural fit or similarities among participants generally favor the cooperative arrangements (see Ward-Peppard 1996).

In an intraorganizational level the business-IS organizational relationship has always been a troublesome, while the two domains differ remarkably from each other with respect to their cultural basis. This so-called cultural gap is often seen as the main variable to explain the troubled relationship between the IS organization and the rest of the organization (Grindley 1992; Ward-Peppard 1996). In practice, the gap can be seen in difficulties to trust, understand and appreciate the other side.

Information system professionals believe that businessmen are not aware of the opportunities IT offers, that there is no personal gain in understanding business better, and that users cannot be responsible for any part of the IS-related development. Similarly, businessmen feel that IS organization is hardly ever contributing to business performance and success, that technical solutions have rarely any practical use, and that IS-related costs often exceed the achieved benefits. (Ward-Peppard 1996; Neo 1988) Accordingly, management is challenged to create appropriate mechanisms for organization-wide value management.

The value management is a mechanism aimed at promoting integration and lowering the communication threshold. Moreover, it helps creating a common language between the integrated parties, thus, fostering the development of shared understanding and trust (Applegate 1994; Hagström 1991). In practice, the senior management may try to bridge the gap through promoting reciprocal education 
programs and by rotating representatives into rival camps, so that gradually both domains gain knowledge of each other. Similarly, they must communicate their support to these projects, and provide examples of the IT possibilities. The successfulness of these mechanisms depend on combining the management of both 'harder' [structures, systems and processes] and 'softer' [symbols, rituals, routines and metaphors] cultural elements into one shared web (Johnson 1992; Land 1992).

The technological development, in turn, focus on creating an open-access interorganizational systems (IOS), where everybody has the freedom to use all information within the network. The network center organizes and manages this infrastructure and builds applications and databases around the shared network relationship approach. The used IOS remain flexible and open in their interfaces across organizational border and, thus, the pressures for change can be adapted in accordance to the external progress and business development.

The IS-education agenda must be extended to cover the newest application development software and internet technologies, so that the IS personnel will gradually learn to master the modern solutions' environment. The on-going training programs support the continuous adaptation process to networking challenge as well as to the interorganizational architecture development (in telecommunications, applications and hardware fields).

The other side in human resource management agenda, continuous renewal challenge, calls for individual skill development and creating the foundation for organizational learning. This decision-making area deals with creating the required human skills and capability for supporting and shaping the business strategy and the final strategic alignment (Venkatraman et al. 1993; Ross et al. 1996). First, the desired skills and capabilities have to be defined to focus resources on the most promising and critical areas for complementing available or potential IS infrastructure and processes. Second, the efforts should be concentrated on capability development within that defined frame, and finally the mechanisms for continuous adaptation are created (e.g. cross-border transfer of applied technologies).

In this way, the human resources are connected to the overall organizational transformation and aligned to the continuos renewal challenge of all networked organizations. When each individual will master the required literacy and tools of both business and IS-domain development, the adaptation takes place more efficiently. Moreover, the individual capabilities play an important role in supporting the aforementioned organizational learning. The common concepts and shared values facilitate a transfer of tacit knowledge across organizational boundaries, and the knowledge and know-how of modern solutions leverage the whole network with other networks.

The learning races and game playing are common tools in helping organizations to find unique operational solutions for novel situations. The network center may act as an innovation market place, where the overall innovation process of the whole network takes place. The races and games help network members to rise above their routine tasks and observe the turbulent environment as a scene of opportunities, not as a minefield of threats. The game playing opens up new horizons for future operations, and can be used as an educational tool in network development. 


\section{Conclusions}

The networked organizations operate under the pressures and influence of reciprocal interdependence, because they have to adjust their actions to many interacting positions and participants. The IS should support collaborative work, which is much less structured than intraorganizational processes and operations. Therefore, networking parties should actively try to discover elements of deep structures in the interaction situation and incorporate this structure to their evolving use of the information systems.

To manage this change, it should ensure that the organizational climate is right and set up for the upcoming challenges. All the stakeholder groups are to be included to the change process and a more democratic and participative culture should replace the traditional command-control structures. In this way, the ownership of the process itself can be spread throughout the network as well as across the business functions.

In conclusion, we argue that the human resource management agenda in networked organizations has two specific areas of concern. The management should 1) coach the network for creating shared understanding and trust, and 2) train members of organization for working in ambiguous settings. However, this work cannot be done alone in a management level with top-down approach, but should include all levels of organization and all stakeholder groups across the interconnected network. In this way, the collaborating parties may create shared vision of the future, and direct the internal training to the most promising fields.

\section{Biography}

Mr. Jukka Viitanen is an assistant professor of the Turku School of Economics and Business Administration. He received his Ph.D. in August 1998 from the Institute of Information Systems Science with a topic of Information Management Strategies in the Global Network Organizations. At the moment, he is serving as a director of the Finnish Institute in Japan, in Tokyo, and engage in researching further Japanese business systems and IS utilization. 